\title{
CREACIÓN DE OBJETOS DIGITALES DE APRENDIZAJE Y SU INCLUSIÓN EN EL REPOSITORIO INSTITUCIONAL ESpacio-UNED
}

\section{(CREATION OF LEARNING DIGITAL OBJECTS AND THE PROCESS OF INSERTION IN ESpacio- UNED INSTITUTIONAL REPOSITORY)}

Luis Zorita

Alicia López Medina

Miguel Latorre

Manuel Blázquez

Elio San Cristóbal

Sergio Martín

Gabriel Díaz

Manuel Castro

Universidad Nacional de Educación a Distancia, UNED (España)

\section{RESUMEN}

El empleo de estándares de etiquetado de objetos digitales es una práctica empleada con profusión para la gestión del conocimiento. Se pretende dar a conocer los trabajos realizados en el seno del Departamento de Ingeniería Eléctrica, Electrónica y de Control de la Escuela Superior Técnica de Ingeniería Industrial de la Universidad Nacional de Educación a Distancia (UNED) en relación con el aprovechamiento y reutilización de los recursos del departamento tales como apuntes, problemas, resoluciones, esquemas, artículos, etc. Estos trabajos de clasificación, etiquetado y organización digital de la información, en una posterior fase de ampliación han sido aplicados para la extracción de recursos procedentes del material publicado en los congresos de Tecnologías Aplicadas a la Enseñanza de la Electrónica. Para almacenar tal cantidad de objetos, la UNED dispone de un repositorio institucional, eSpacioUNED, sobre el cual se ha realizado un proceso de volcado masivo de objetos y archivos asociados de metadatos.

Palabras clave: objetos digitales, objetos de aprendizaje, repositorio, reusabilidad, integración de servicios.

\begin{abstract}
The use of marking and labeling standards in digital objects is a process widely implemented for managing knowledge. The presented paper aims to explain the works developed from the
\end{abstract}


Electronics and Computer Engineering Department in UNED in the way to take advantage and reuse the department resources such as annotations, problems, solutions, schemes, articles, etc. The works have been focused through classification, labeling and digital organization of information, having been extended in a subsequent stage of extraction and management of those digital objects susceptible to be extracted from the publications of the papers presented in TAEE conferences. In order to storage such an amount of digital objects, the university institutional repository, eSpacio-UNED has been selected to load both, learning objects and metadata associated files.

Keywords: digital object, learning object, repository, reusability, services integration.

La enseñanza apoyada en las TIC está asociada a un número elevado de contenidos digitales. Esta fuente de referencia cobra una gran importancia en el ámbito de la Ingeniería dada la diversidad de herramientas para la creación y observación de experimentos. Desde presentaciones sobre los conceptos en estudio, hasta simulaciones mediante computador con distintos grados de complejidad, todos estos recursos multimedia interactivos facilitan el análisis de sistemas reales comparando su comportamiento con aproximaciones sobre modelos teóricos de sus componentes.

Este planteamiento de aprovechamiento de contenidos digitales ha sido el punto de partida de los trabajos que aquí se presentan y que comenzaron con la extracción y gestión de los recursos digitales de un departamento para su empleo posterior con múltiples propósitos, y que siguieron con la extracción de objetos digitales procedentes de congresos profesionales sobre docencia de la Electrónica. Finalmente se presenta el repositorio institucional eSpacio-UNED donde se han almacenado todos los objetos digitales junto con sus metadatos como elementos de definición.

\section{RECURSOS DIGITALES ABIERTOS PARA EL APRENDIZAJE DE LA ELECTRÓNICA}

En los entornos de trabajo sobre computador confluyen por este motivo múltiples formatos para la codificación de la información. No obstante, la reutilización de los materiales mencionados anteriormente en otros contextos educativos implica un coste de modificación muy significativo. La reorganización de los archivos en unidades conceptuales con diferente granularidad, la adaptación de las experiencias según el perfil del estudiante (e.g. edad, curso académico) y su velocidad de progreso, o la autoevaluación de las actividades no se contemplan en la autoría. Para cubrir esta necesidad surge el modelo de referencia de objetos SCORM basado en estándares abiertos orientados a la Web para la descripción y el empaquetado de 
recursos digitales educativos (ROU, 2004). A partir de dicho estándar se propició el desarrollo de los entornos para la gestión del aprendizaje (LMS) existentes en la actualidad, los cuales facilitan al instructor la estructuración de las creaciones destinadas al estudiante según un enfoque constructivista.

Siguiendo esta evolución de la enseñanza a distancia, el Departamento de Ingeniería Eléctrica, Electrónica y de Control de la UNED ha publicado el catálogo de ejercicios relacionados con las materias de Ingeniería Electrónica aplicando estas tecnologías. En un primer estadio se reorganizaron los archivos en una ruta única para su tratamiento posterior. La clasificación de los ficheros originales guardados en soporte óptico arrojó los siguientes resultados:

- Electrónica Analógica

- Electrónica Digital

- Teoría de Circuitos

- Física para Informática

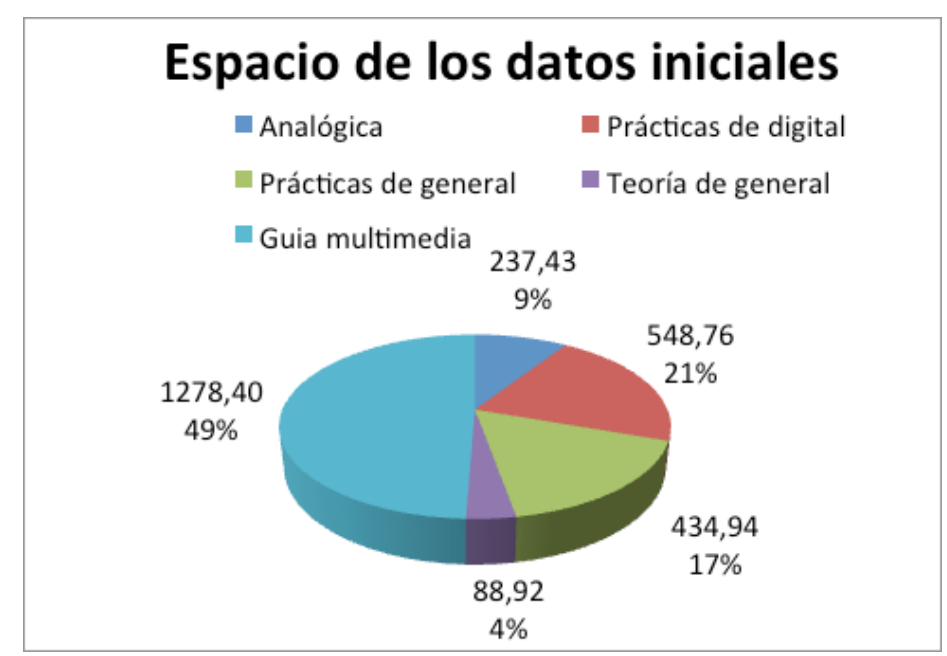

Figura 1. Distribución de objetos digitales extraídos del DIEEC

Encontrar un documento concreto dentro de la estructura imponía una serie de restricciones estrechamente vinculadas tanto a la búsqueda por nombres de archivo, como a las relaciones simples dentro de la jerarquía de directorios. Para superar estas limitaciones se hace necesario dotar a los documentos de la información 
adicional necesaria: título del documento, tema, disciplina o curso académico. El procedimiento ha consistido en asignar descripciones con una sintaxis/estructura accesible por cualquier repositorio digital o conjunto de colecciones de objetos, LMS y buscadores de Internet (incluidos buscadores especializados como Google Académico). Estas descripciones, denominadas metadatos (MAR, 2006), constan de una serie de bloques para caracterizar el ciclo de vida, detalles técnicos de uso, aspectos educativos, autoría, relaciones con otros objetos, etc.

Un requisito esencial en el procedimiento previo es la generación automática de la mayor parte de los campos con sus valores correspondientes, asegurando en todo momento la conformidad de los resultados con un esquema. Esta verificación se realiza con una herramienta para validar los metadatos, la cual coteja la lista de elementos frente a una plantilla estándar. En este caso, el formato asignado es LOM v1.o en su variante sobre el lenguaje XML. Con el estándar designado hemos de cumplimentar dos fases: extracción de los valores y generación de los metadatos.

La primera etapa consiste en adquirir la información con la cual rellenar los valores para cada uno de los descriptores. Dado que las actividades acompañan a una bibliografía, los libros de texto facilitan todos los detalles con una serie de tablas en los anexos. Por lo tanto, la extracción de las tablas resuelve la última parte. En la práctica esta fase queda completada con la adquisición de datos mediante un programa de reconocimiento óptico de caracteres de código abierto (gOCR).

Dentro de la segunda etapa partimos con todas las características disponibles en versión digital. Un script combina cada uno de los elementos con su valor dentro de la plantilla. El resultado conseguido se plasma en un conjunto de ficheros XML con el mismo nombre que las actividades descritas. A través del emparejamiento de los recursos con sus atributos se permite una exploración instantánea por categorías, dejando así en un segundo plano el filtrado de extensas listas de coincidencias con búsquedas manuales por palabras clave. Este cambio implica favorecer el descubrimiento de la información según la temática de interés. El acceso se realiza mediante un catálogo destinado a este propósito con cualquiera de los navegadores Web actuales.

Los pasos necesarios para manipular los nuevos objetos se simplifican respecto a sus contrapartidas locales. Una vez localizado el documento de interés, éste puede incorporarse en un LMS de otra institución, modificarlo con un editor especializado (e.g. ReLoad Editor), o compartirlo con otros usuarios. Con los últimos avances en las aplicaciones Web aparecen como operaciones destacadas la edición directa en un LCMS, o, la simulación directa de circuitos Spice en CircuitLab. Esta interacción 
directa elimina la administración documental sobre los archivos y carpetas que los contienen.

En definitiva, tras el proceso descrito se han llegado a manejar cifras de objetos digitales directamente extraídos de los materiales del departamento tal y como se representan en la siguiente tabla 1 :

\begin{tabular}{|c|c|c|c|}
\hline \multirow{4}{*}{$\begin{array}{c}\text { Disciplinas } \\
\text { tratadas } \\
\text { (68 temas) }\end{array}$} & \multicolumn{3}{|c|}{ Analógica } \\
\hline & \multicolumn{3}{|c|}{ Digital } \\
\hline & \multicolumn{3}{|c|}{ Teoría de circuitos } \\
\hline & \multicolumn{3}{|c|}{ Física para informática } \\
\hline \multicolumn{3}{|c|}{ Tipos de actividades } & Resultados \\
\hline Enunciados & Resueltos & Prácticas & Total de metadatos \\
\hline 158 & 886 & 177 & 1896 \\
\hline Propuestos & \multicolumn{2}{|c|}{ Teóricos } & Total de archivos \\
\hline 405 & \multicolumn{2}{|c|}{270} & $7633(188 \mathrm{M})$ \\
\hline
\end{tabular}

Tabla 1. Contenidos procesados y resultados finales

\section{LA GESTIÓN DEL CONOCIMIENTO GENERADO EN LOS CONGRESOS DE TECNOLOGÍAS APLICADAS A LA ENSEÑNANZA DE LA ELECTRÓNICA}

Una de las aplicaciones más extensas llevadas a cabo en el seno del proyecto de Reutilización de Objetos Digitales (ARR, 2006) ha sido la recopilación de los contenidos publicados en los congresos TAEE desde su primera edición de 1994 hasta su última edición en 2012. TAEE (Tecnologías Aplicadas a la Enseñanza de la Electrónica) es un proyecto cuyo ánimo es el de promover la aplicación de las Tecnologías a la enseñanza de la Electrónica. Entre otros medios, TAEE emplea los congresos para que docentes y profesionales tengan un espacio de divulgación de trabajos relacionados con la Ingeniería, la Electrónica y la metodología Docencia.

\section{Organización general del proyecto de objetos TAEE}

El alcance del proyecto presentado en este epígrafe ha consistido en la creación semiautomática de objetos educativos (OBE, 2009) de TAEE, lo que ha supuesto el tratamiento de más de 1.000 publicaciones de las que se han extraído elementos y 
objetos constituyentes de las publicaciones. Este proyecto, por su complejidad, se ha dividido en etapas, que de forma general se nombran como:

- Homogeneización de la documentación TAEE.

- Extracción de objetos educativos desde cada una de las publicaciones.

- Recopilación de la información definitoria de cada publicación.

- Creación de archivos de metadatos con la aplicación estándares de marcado.

- Asociación del objeto educativo a su archivo de metadatos.

Cada una de estas etapas, persigue la creación de un fondo documental con posibilidad de acceso a objetos de diversa granularidad, tamaño, composición y utilidad, gracias a la información contenida en los metadatos asociados a cada objeto digital de aprendizaje u objeto educativo. Es decir, mediante los metadatos, que esencialmente son datos que describen la información que se desarrolla en un determinado objeto digital de aprendizaje tendría que ser posible un reconocimiento de la documentación sin tener que acceder a ella, o mejor dicho, los metadatos tendrían que ofrecer un conocimiento superficial de la documentación buscada para el reconocimiento de su utilidad. En esencia, la implementación del lenguaje de etiquetado en archivos asociados a la documentación permitiría identificar y ayuda a seleccionar la información de forma óptima.

Además, una de las propiedades primordiales exigibles a los ODA es la propiedad de reusabilidad. Por reusabilidad, se entiende la capacidad de utilización integra de un objeto determinado para su incorporación en un contexto que no tiene porque ser del mismo ámbito que el contexto original del objeto. A modo de ejemplo, se puede pensar en un documento digital que contenga la descripción textual y gráfica de un transistor genérico de silicio en el ámbito de la realización de prácticas de electrónica en los primeros cursos de Ingeniería de una determinada Escuela Universitaria.

Si se extrae dicho documento de este contexto y se convierte en objeto digital de aprendizaje (ODA), por sí solo es una unidad que perfectamente puede incorporarse en un curso cuya finalidad sea la exposición teórica de los transistores en el ámbito de un curso de formación electrónica para alumnos de Bachillerato o en un curso de formación para personas desempleadas. No obstante, la formación y creación de objetos de aprendizaje es una actividad compleja que conlleva una serie de pasos de planificación, entendiendo la complejidad como que existen dificultades dada la 
variable complejidad de los objetos, su composición interna, su estructuración, etc. Este conjunto de propiedades se unifica en el término de granularidad de los objetos digitales.

Conjuntamente con la granularidad, que atiende a un concepto cuantitativo, se ha de tener en cuenta las características cualitativas, es decir, las que contemplan la heterogeneidad en los elementos componentes de un objeto digital, que dará lugar a la existencia de unidades con formatos tan diversos como textos, gráficas, fotografías, tablas de datos, etc.

Dado, pues, que el principal objetivo de los lenguajes de marcas y de los metadatos reside en su utilidad para definir información a la que hacen referencia, sería apropiado que esta metainformación fuera lo más concreta y definitoria posible, ya que los metadatos suministran los detalles a los medios que utilizan los buscadores de Internet para localizar la información ante una petición de consulta.

De forma extensiva, los servicios que se ofrecen con el uso de metadatos alcanzan aplicaciones como la formación de bases de datos con la información de los metadatos, el control de flujo de información e incluso la creación de algoritmos de compresión más eficaces en función del tipo de información que ofrezcan los metadatos de unos datos asociados.

En esta línea, el marcado de las características y constitución de un objeto digital que tenga multitud de elementos componentes será fundamental para el éxito de su reutilización. Esto se llevará a cabo con una escrupulosa estructuración de las relaciones entre elementos principal y componentes, en la que los metadatos jugarán un papel esencial tanto en la definición de cada objeto y de cada componente como en el establecimiento de las relaciones entre ellos. Más adelante, se definirán los estándares de marcado y etiquetado de objetos digitales y específicamente, los metadatos indicados para establecer las relaciones entre ellos.

Para minimizar la complejidad comentada, se considera una buena práctica que los ODA cubran un único objetivo de aprendizaje. Para lograrlo deben mantener independencia del contexto y no requerir de otros recursos, es decir, que sean autosuficientes. Con esta filosofía, la definición de la información mediante los metadatos gana en precisión, dado que tal y como se ha definido en el anterior apartado, los metadatos serán el conjunto de atributos o elementos necesarios para describir suficientemente un recurso a modo de primer acercamiento con el objeto. 
Traduciendo de la obra "Professional XML Metadata", los autores consideran (AHM 2001, p.86) "que los metadatos basados en tecnología XML son un elemento clave para la administración de repositorios digitales, pudiéndose además llevar a cabo el intercambio de información y de contenidos, entre plataformas y entre repositorios, de forma transparente para el usuario", razón por la cual la mayoría de los esquemas de metadatos propuestos por diversas iniciativas, están basados en tecnología XML.

Esta tecnología sigue las recomendaciones del $\mathrm{W}_{3} \mathrm{C}$ respecto al uso del lenguaje $\mathrm{XML}\left(\mathrm{W}_{3} \mathrm{C}, 2008\right)$ en las que se fijan claramente los objetivos de diseño del lenguaje que se citan a continuación:

- XML debe ser utilizable directamente sobre Internet.

- XML debe soportar una amplia variedad de aplicaciones.

- XML debe ser compatible con SGML.

- Debe ser fácil escribir programas que procesen documentos XML.

- El número de características opcionales en XML debe ser mantenido en un mínimo, idealmente cero.

- Los documentos XML deben ser legibles por un humano y razonablemente claros.

- El diseño de XML debe ser preparado rápidamente.

- El diseño de XML debe ser formal y conciso.

- Los documentos XML deben ser fáciles de crear.

- La brevedad en la marcación es de mínima importancia.

\section{El tratamiento de los objetos educativos reutilizables mediante estándares de metadatos existentes}

Entre las múltiples aplicaciones de los metadatos, el manejo de objetos educativos para su reutilización es un medio muy eficaz de interrelacionar información cuyo destino no solamente es aquel para la que fue creada, sino que puede ser incluida 
en múltiples ámbitos y entornos. Esta idea tan sencilla, es realmente revolucionaria dentro del ámbito del e-Learning y la docencia a distancia.

Tal y como se ha indicado en anteriores apartados, los metadatos no solamente ayudan a definir la información de referencia sino que además proponen un modelo de relación con otros objetos o unidades de información. Dada la amplitud del planteamiento, existen infinidad de escenarios donde ubicar un objeto educativo, de forma que para acotar o al menos, organizar los espacios de aplicación del objeto, es adecuado el uso de estándares de aplicación.

En esta línea, dentro del ámbito de la documentación existen dos corrientes de utilización, una primera basada en la organización de la información y la documentación propia del ámbito de la Biblioteconomía y una segunda que enfoca el etiquetado de la información para su uso como objeto reutilizable. En el primero de los casos citados, la organización de la información y la documentación tiene por estándar Dublín Core (DCM, 2013).

En el segundo de los casos planteados, la utilización de la información como elementos u objetos reutilizables, es el IEEE (Institute of Electrical and Electronics Engineers) quien ha desarrollado el estándar LOM, Learning Object Metadata (LOM, 2002). Como consecuencia, todos los objetos educativos etiquetados en el proyecto han tenido una triple definición según los citados estándares Dublin Core e IEEE-LOM, pero también mediante una estructura de metadatos llevada a cabo expresamente bajo criterios de utilidad en el entorno de la institución TAEE.

\section{El proceso de individualización de los objetos TAEE}

Bajo un punto de vista estrictamente formal, el proceso de atomización de la documentación TAEE en objetos reutilizables consiste en la separación y regeneración física de un archivo que contiene únicamente los datos referentes a una única ponencia. Una vez realizada esta separación se procede a la visualización completa del contenido del archivo y se van extrayendo uno a uno todos los elementos internos que resulten útiles como objetos reutilizables.

Atendiendo al término de granularidad y partiendo del objeto de mayor volumen, la publicación del conjunto de comunicaciones de un congreso dado, se ha procedido a la separación de cada unidad de ponencia o artículo, denominado ODA nivel 1. En un segundo proceso de revisión y examen de los ODA nivel 1, se ha procedido a la extracción de aquellos elementos de información contenidos en estos, esto es, los ODA nivel 2. 
Tras el proceso de extracción de objetos digitales, se obtuvieron más de 1.000 objetos ODA nivel 1 y de ellos han surgido casi 6.000 objetos ODA nivel 2. Los primeros han sido tratados como documentos electrónicos manteniendo su formato original en PDF, tras el proceso de escaneado en el caso de los primeros congresos. Por otra parte, los ODA nivel 2 han tenido un tratamiento que se ha adecuado a su naturaleza gráfica, electrónica o textual.

El volumen de objetos extraídos ha justificado la creación de un sistema de codificación de documentos, lo más inteligente posible desde el punto de vista de reconocimiento de los mismos. Para elaborar el sistema de codificación se han impuesto desde el origen, criterios que facilitan su situación y emplazamiento en una estructura de ficheros.

De todas las alternativas para implementar una codificación eficiente, se ha decidido no aplicar criterios taxonómicos sino criterios de localización temporal. Esta inclinación surge aprovechando una constante en todos los congresos de TAEE desde su fundación, consistente en la división de los congresos en jornadas y las jornadas en sesiones.

Cada sesión distribuye exposiciones y ponencias de forma acotada en franjas temporales.

El sistema de codificación general de la documentación de SCGD-TAEE mostrado en la figura 2, tiene por tanto una estructura muy sencilla pero a la vez muy potente dado que de por sí, contiene los primeros metadatos que interesan acerca de la localización temporal en origen del documento definiendo año, sesión y situación correlativa de la ponencia. Este código, definido por una cadena de caracteres numéricos y alfabéticos, no solamente define estos metadatos sino que será clave en las posteriores manipulaciones del resto de metadatos asociados a los objetos de aprendizaje.

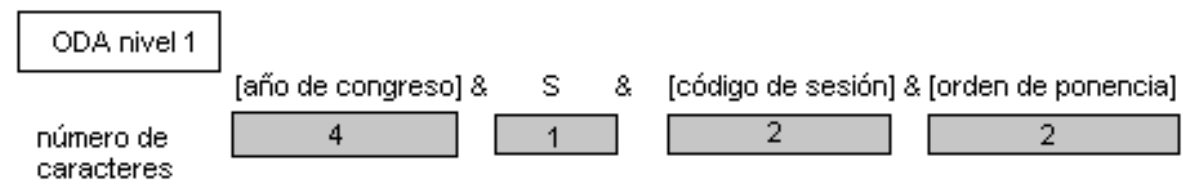

ODA nivel 2

número de caracteres [código padre ODA nivel 1] \& [código tipo hijo] \& [código orden hijo]

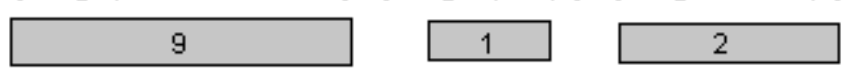

Figura 2. Codificación aplicable a los documentos TAEE 
Como se observa en la figura 2, se distinguen dos tipos de códigos: los aplicables a los objetos tipo ponencia u ODA nivel 1 y los aplicables a sus elementos internos u ODA nivel 2. En el primer caso, el código posee cuatro caracteres correspondientes a año de congreso, con lo que su situación temporal en el conjunto está asegurada, sobre todo a la hora de manipular archivos en paquetes anuales. Se sitúa a continuación un carácter fijo "S" que distinguen los dos siguientes caracteres que corresponden con el nombre clave que se ha asignado a la sesión en la que se incluye la ponencia. Finalmente se añaden dos caracteres numéricos más que indican el número de orden de la ponencia en el seno de la sesión.

En el caso de los objetos ODA nivel 2, lógicamente el código se extiende, dado que sus nueve primeros dígitos corresponden al código que se haya asignado al objeto del que proceden u objeto padre. Se le añadirán a continuación tres dígitos más, uno para indicar la naturaleza del objeto y dos más que ofrecen un número de orden de aparición en el documento. Los elementos encontrados y que son susceptibles de convertirse en objetos de aprendizaje de nivel 2 son diagramas, esquemas eléctricos, fotografías e ilustraciones, gráficos, tablas de datos, textos planos, códigos fuente de programas y instantáneas de páginas web o de ventanas de aplicación.

\section{Utilidades del código de documentación}

Según lo indicado con anterioridad, además de ordenar de forma organizada los documentos individualizados que se generan, la utilidad del código de documentación asignado facilita la asociación de todos los archivos que bajo diferentes formatos se refieran a un documento u objeto dado. A modo de ejemplo, el código 2006S1Ao4, indicativo de una ponencia del congreso de 2006, de la sesión $1 \mathrm{~A}$ con orden 04 de exposición, se nombrará con extensión " $p d f$ ” cuando se trate del documento de los contenidos de la ponencia, o con extensión " $x m l$ " cuando se trate del fichero de metadatos, o con extensión "html" cuando se trate de ofrecer información sobre el documento al usuario Web.

Llegado a este punto, al finalizar la fase de extracción documental, cada documento ha sido clasificado y normalizado mediante la aplicación de un sistema de codificación unificada basado en la propia estructura de los congresos TAEE, que implica la denominación formal de todos los archivos relacionados con el objeto educativo mediante el mencionado código inteligente. Las ventajas que ofrece, por tanto, el código documental son:

- Proporcionar, de forma natural, los primeros metadatos como el año de congreso, la sesión de la ponencia y el orden de exposición de la misma. 
- Permitir la relación entre los objetos de forma jerarquizada con criterios de descendencia de objetos de nivel 2 que resulten del documento digital original.

- Facilitar la búsqueda en bloque de los archivos que se asocien a una misma ponencia, a una determinada sesión o de forma general a un congreso dado.

- Facilitar la localización de los ficheros relacionados con determinado objeto educativo.

Esta fase finaliza con el posicionamiento de cada objeto educativo en la estructura general de ficheros unificada, diversificando cada objeto en cada carpeta anual y alojando éstos en el subdirectorio previsto. Esta organización facilita el acceso a los objetos digitales utilizando direccionamiento relativo, lo que da mayor versatilidad al código utilizado para su acceso.

\section{La estructura de metadatos}

Los objetos de aprendizaje proceden de diferentes fuentes, siendo cada una de ellas un compendio de publicaciones derivadas de cada congreso. Cada comité organizador propone a los autores la preparación de un documento que describe su trabajo, mediante un documento de plantilla que sirve a los autores como guía para la inclusión de elementos textuales y gráficos que componen su obra. En el análisis de todas las ponencias de los congresos, se han encontrado multitud de divergencias en la aplicación de las normas de la plantilla propuesta en cada congreso, con lo que ha sido necesario realizar un proceso de relectura y homogeneización de formatos textuales de las cadenas de caracteres extraídas de forma automática.

Los documentos XML de metadatos que definen las características de tal diversidad de objetos de aprendizaje tiene un formato común para todas ellas. Para homogenizar las cadenas de caracteres se han propuesto, de forma básica, unas sencillas normas, fundamentalmente referidas a:

- Utilización de caracteres en minúscula para textos generales.

- Utilización de caracteres con letra capital para nombres propios.

- Revisión exhaustiva y corrección de errores ortográficos.

- Supresión de caracteres de saltos de línea, tabulaciones y demás controles textuales. 
- Utilización del caracter especial “;” para la concatenación de datos múltiples en un mismo campo.

Con la aplicación de esta normativa, las cadenas de caracteres que pasarán a formar parte de la estructura de metadatos, han sido volcadas de forma manual o de forma semiautomática en una hoja de datos, empleándose para ello una hoja de cálculo. La selección de esta herramienta se ha basado en su versatilidad y accesibilidad, aunque en realidad los criterios de mayor peso han sido su capacidad para proporcionar una estructura cartesiana de organización textual y la propiedad natural de contención de texto carente de formato.

Con posterioridad, en el proceso de volcado de datos se ha utilizado herramientas incorporadas en la aplicación para la búsqueda y sustitución de cadenas de caracteres, como elementos de corrección rápida de los textos. Además, la hoja de cálculo dispone de módulos de programación en lenguaje mediante los cuales se ha procedido a la elaboración y ejecución de programas a medida para el tratamiento de textos. Ha sido de especial utilidad el uso de estos programas para la separación de metadatos que contenían en origen múltiples referencias como por ejemplo, el metadato que soporta los nombres de los autores de las obras o cuando una multiplicidad de organismos participa en una publicación o el campo destinado a alojar las palabras clave. Cuando se ha tenido que formar el metadato con las referencias múltiples se ha utilizado el carácter ";" para la separación de los mismos.

Esta norma permitida en la estructura de metadatos específica de TAEE, no es recomendable por los sistemas estándar de metadatos, como IEEE-LOM o Dublin Core, con lo que ha sido necesaria la separación de cadenas de caracteres con entidad propia. Aquí es donde ha jugado un papel importante la elaboración de software para la separación y manipulación de datos simples contenidos en cadenas de caracteres múltiples.

Una vez que se han eliminado heterogeneidades y se han aplicado las normas citadas con anterioridad en diversas hojas de cálculo, se ha aprovechado la capacidad para la exportación de datos de forma rápida, eficaz y sencilla a bases de datos con conectividad ODBC. Este tipo de bases de datos son de tipo relacional con lo que se ha podido enlazar tablas mediante el código documental. Así, para la manipulación masiva de datos, se han programado consultas en la propia base de datos a fin de generar tablas derivadas que contengan la estructura de datos deseada.

Ya se ha comentado con anterioridad acerca del empleo de sistemas estándares de metadatos Dublin Core e IEEE-LOM para crear estructuras de metadatos 
alternativas. De ambos, LOM es el estándar que más se adapta a las necesidades de acopio de metadatos en TAEE. No obstante, para evitar ciertos metadatos específicos propios del tipo de objetos que se presentan en los congresos TAEE, se ha desarrollado una tercera vía de definición, la estructura de metadatos TAEE. Esta estructura se ha diseñado desde un concepto arbóreo tal y como se representa en la siguiente figura 3:

\section{ESTRUCTURA DE METADATOS}

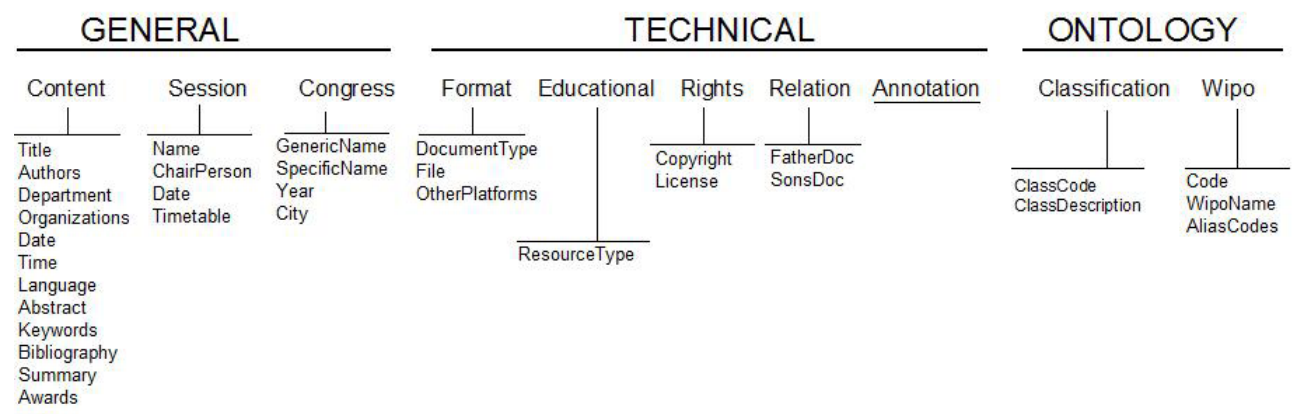

Figura 3. Estructura TAEE de metadatos

La estructura se compone de tres grandes ramas o bloques de metadatos envueltos en las respectivas etiquetas General, Technical y Ontology, siguiendo una estructura más compacta que la propuesta por el estándar IEEE-LOM, que plantea nueve bloques de metadatos. Esta es la primera gran diferencia con el estándar aunque mantiene cierta similitud que permite trazar compatibilidades entre los sistemas.

\section{La Generación automática de ficheros XML de metadatos}

Dada la ingente cantidad de metadatos a manipular, primero debido a la generación de metadatos según tres sistemas diferentes y segundo, por el elevado número de objetos digitales a manejar, se ha desarrollado una aplicación específica de creación de documentos XML, la aplicación lanzadera de metadatos. El esquema genérico que se ha seguido en la elaboración de la aplicación de generación de archivos XML, o Aplicación Lanzadera de metadatos, sigue el esquema proporcionado por el gráfico de la figura 4, en el que se transforman los datos contenidos en cada registro de una base de datos relacional en ficheros XML. 


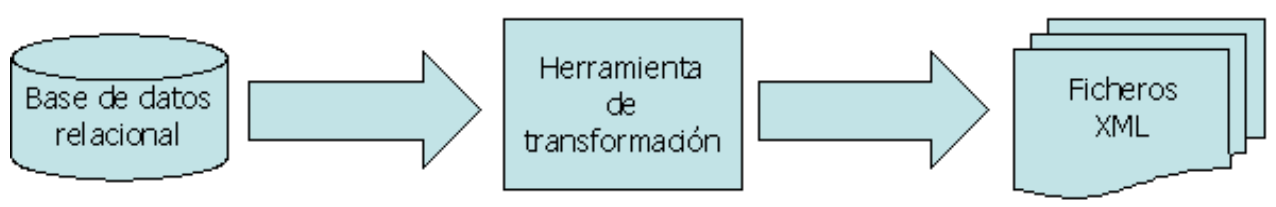

Figura 4. Esquema genérico de la Aplicación Lanzadera de metadatos

Como primer acercamiento al proceso de creación de ficheros XML de metadatos, la aplicación que ha llevado a cabo esta labor, la Aplicación Lanzadera, tiene como origen de datos, todos los metadatos organizados en las tablas de una base de datos. Esta base de datos, como se ha descrito con anterioridad, se ha construido con una estructura adecuada a los diferentes formatos necesarios para cada modelo de fichero XML: TAEE, LOM o Dublín Core. Por consiguiente, la entrada de datos de la aplicación Lanzadera será la citada base de datos. Esto ofrece una solución de partida abierta a cualquier tipo de proceso, ya que, como se verá más adelante, el programa se adaptará de forma universal a cualquier base de datos, sea cual sea su extensión y sea cual sea su estructura.

En esencia, tal y como se desarrolla en los siguientes apartados, el programa Lanzadera se encargará de abrir la base de datos, conectar con la tabla donde residan los metadatos y extraer el contenido de los campos que componen la tabla y que constituyen los propios metadatos. Para ilustrar el proceso general de funcionamiento se ha representado en la figura 5 un diagrama de bloques que define el funcionamiento en el flujo de datos de la aplicación Lanzadera. 


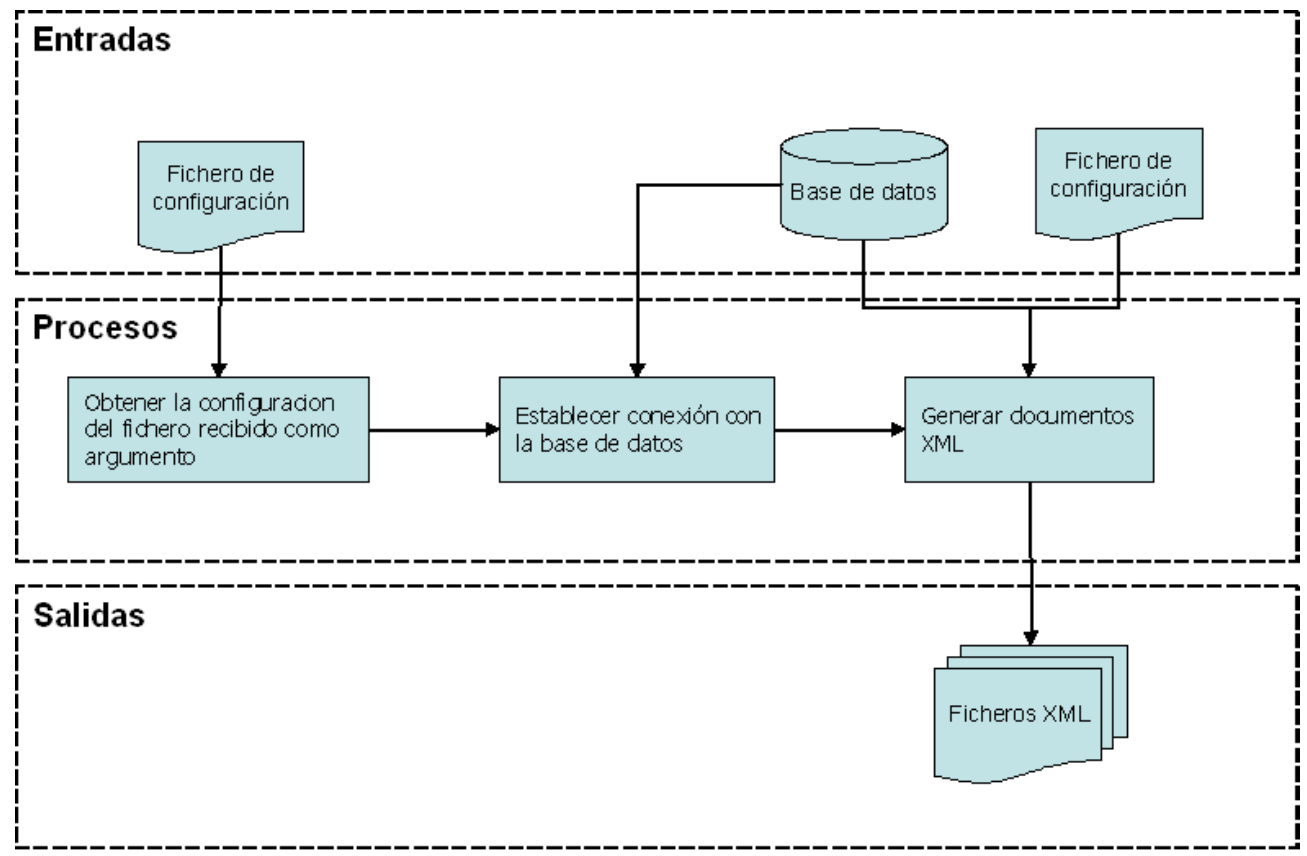

Figura 5. Diagrama de bloques del funcionamiento de la aplicación Lanzadera

En esta figura se observan los componentes de la aplicación, la cual dispone de un fichero de configuración y la propia base de datos relacional como elementos de entrada y la plantilla de metadatos como elemento de salida. La organización de los archivos se encuentra dividida en directorios de igual forma, esto es, existirá un directorio donde se ubiquen los ficheros de configuración, un directorio donde se encuentre el código fuente JAVA y un directorio donde se ha previsto la ubicación de los archivos XML que se generen en el proceso de creación. La estructura de archivos de la aplicación lanzadera dispone de un directorio de configuración que contiene además plantillas de archivos XML sobre las que se sobrescriben de forma automática los metadatos. Asimismo se ha previsto un directorio de librería para poder configurar el empleo de bases de datos OCDB, JDCB o del entorno Oracle.

El proceso de generación de archivos XML de metadatos, representado en la figura 6, alude a un proceso recursivo de búsqueda e imposición de valores desde su origen en la base de datos hasta su ubicación final en la plantilla correspondiente, con lo que la función de coste del programa es lineal y dependerá del número de registros que se quieran reproducir en ficheros XML. 


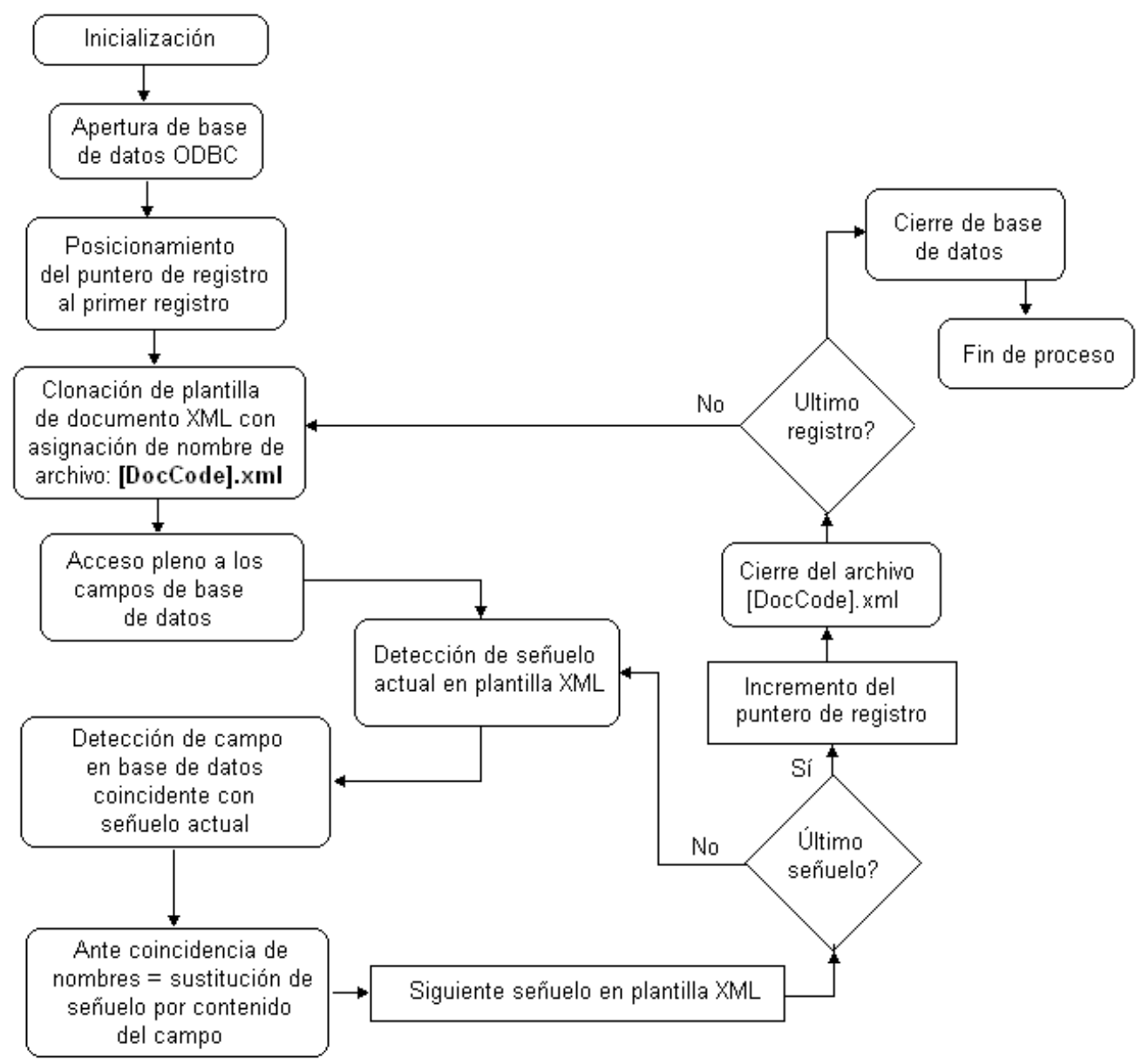

Figura 6. Algoritmo gráfico del programa lanzadera

Dentro de la base de datos, la tabla principal que contiene los datos principales de cada archivo de metadatos dispondrá de un campo, el código documental que actúa como elemento clave en la ejecución del programa y como dato que dará nombre a cada archivo de metadatos que se genere.

La aplicación en su ejecución, en primera instancia, procede a la clonación del nuevo archivo desde la plantilla con el nuevo nombre, quedando disponible para la modificación de sus contenidos. Se produce entonces el inicio de la lectura de los valores del registro actual, guardando los mismos en un conjunto de variables dotadas de dos elementos: el nombre del campo y el valor del mismo. Esto supone 
por tanto, el acceso pleno a los valores de los campos de la base de datos. Hay que reseñar que en este paso reside el carácter abierto de la aplicación, dado que no se accede a nombres concretos de campo, sino que pueden definirse como el usuario quiera. De esta forma, queda a discreción del usuario la denominación de cada uno de los campos de la tabla.

Memorizados nombres y valores de campos, se accede a la plantilla. En este caso, se utiliza la fuerza bruta para la modificación de los contenidos de la plantilla. Dicha plantilla que se reproduce en siguientes apartados, está estructurada en código XML utilizando para ello, las etiquetas definitorias. La diferencia entre la plantilla y un archivo XML, reside básicamente en que el valor de metadato ubicado en cada etiqueta corresponde con la concatenación textual del nombre del campo precedido del carácter “\$”, indicativo del carácter de señuelo sustituible del nombre impuesto por el del valor que porta las variables de lectura de la base de datos. Por ejemplo, si el metadato del título del objeto digital se encuentra en el campo denominado"Title", el código existente en la plantilla XML será “\$Title”.

Dada la coincidencia entre la denominación de campos de la base de datos y los señuelos de la plantilla XML, la aplicación lanzadera tan solo tiene que realizar un barrido en profundidad de toda la plantilla, eliminando los señuelos e insertando los metadatos en su lugar. Por supuesto, el carácter de plena adaptación de la aplicación a cualquier definición de campos de bases de datos no es gratuito, dado que el sistema exige total coincidencia de nombres entre los campos de la base de datos y los señuelos dispersos en la plantilla. Para mayor seguridad en la integridad de los datos, en la plantilla se ha de disponer de un listado de campos existentes en la base de datos que se han de utilizar posteriormente en los señuelos.

En caso de utilizar la plantilla para otra tabla, cuando se inicie un proceso de utilización de la aplicación Lanzadera, será necesario disponer de todos los nombres de los campos de la base de datos de origen que se utilicen y proceder al cambio de nombre, tanto del listado inicial de la plantilla como en cada uno de los señuelos. Si no existiera total coincidencia de nombres, el programa en su ejecución no encontraría donde ubicar el valor del campo de definición errónea en la plantilla, no pudiendo modificar el señuelo existente. El proceso de búsqueda y sustitución de los señuelos acaba ante la detección del último de ellos, con lo que se procede al cierre y constitución del nuevo fichero XML, que es ubicado en el directorio de salida correspondiente. El programa efectúa entonces un incremento en el puntero de registro, procediendo a realizar las mismas acciones sobre los datos contenidos en los campos del siguiente registro de la base de datos. 
Este proceso recursivo se lleva a cabo mientras exista un registro dotado de datos y finalizará ante la detección del primer campo vacío. El programa, entonces, procederá a cerrar entonces la base de datos, concluyendo de esta forma el proceso de creación de ficheros XML. Por tanto, y resumiendo las funciones y acciones que la aplicación Lanzadera lleva a cabo sobre las plantillas, se puede indicar que la acción principal consistirá en ejecutar la orden de Generación de documentos XML, mediante la ejecución de los siguientes pasos:

- Carga de la plantilla XML en memoria.

- Consulta la tabla principal de la base de datos.

- Para cada registro de la tabla principal:

- Genera una copia de la plantilla.

- Obtiene los nombres de los campos del registro previstos en el código fuente de la plantilla y carga en memoria los valores de los mismos.

- Rellena en la primera etiqueta de la copia de la plantilla cada referencia al campo por su valor en el registro actual como Proceso de Relleno.

- Elimina de la copia de la plantilla las etiquetas de registro.

- Almacena la copia de la plantilla ya rellena en un fichero en la carpeta de salida.

El proceso denominado de relleno, en detalle, sigue particularmente los siguientes pasos:

- Para cada atributo de la etiqueta:

- Rellena cada referencia a un campo de base de datos en el valor del atributo, por su valor para el registro actual.

- Si el valor almacenado dentro de la etiqueta (<etiqueta $>$ valor $</$ etiqueta $>$ ), es una referencia, la rellena con el valor del campo de base de datos para el registro actual.

- Para cada subetiqueta: 
- Si la subetiqueta no es una etiqueta de tipo elemento repetitivo, vuelve a ejecutar el Proceso de Relleno sobre esa subetiqueta.

- Si la subetiqueta sí es una etiqueta de tipo elemento repetitivo:

$>$ Consulta la tabla secundaria.

Para cada registro de la tabla secundaria:

- Genera una copia de la etiqueta repetitiva.

- Obtiene los nombres de los campos del registro que tiene que sustituir en la etiqueta repetitiva, y carga en memoria los valores de los mismos. Esta carga se mantiene mientras esté dentro de la etiqueta repetitiva.

- Vuelve a llamar al Proceso de Relleno sobre la etiqueta repetitiva.

- Añade la etiqueta repetitiva ya rellena, a la copia de la plantilla.

Elimina de la copia de la plantilla las etiquetas de registro que configuran la etiqueta repetitiva.

Llegado a este punto se dispone de una estructura de archivos asociados a un objeto digital de aprendizaje, que contiene al mismo y a sus archivos de metadatos. Esto permite su empleo en todo tipo de plataformas, especialmente aquellas que utilizan objetos SCORM. No obstante, y sin perjuicio de lo anterior, finalmente se ha empleado esta estructura para la creación de la web global de TAEE y para la inserción de la estructura en el repositorio institucional de la Universidad Nacional de Educación a Distancia, eSpacio-UNED.

\section{EL REPOSITORIO INSTITUCIONAL ESPACIO-UNED}

El repositorio eSpacio-UNED es el fruto de la búsqueda de una solución tecnológica, capaz de almacenar y preservar objetos digitales con componentes heterogéneas (componentes ZIP, XML, TXT, etc). Como segundo objetivo, el repositorio dispone de API para gestión e ingesta masiva de esos objetos. De forma adicional, cumple las siguientes características: 
- Dispone de una ontología capaz de establecer relaciones de inclusión, pertenencia o equivalencia capaz de organizar las relaciones existentes entre los objetos digitales.

- Tiene un punto de acceso sparql para poder integrar los objetos digitales dentro de la web semántica.

Este repositorio está basado en un software open source con licencia "Creative Commons Attribution-Share Alike 3.o Unported License" llamado FEDORA y cuyas características más reseñables son:

- Almacenar cualquier tipo de contenido junto con sus metadatos.

- Capaz de albergar millones de objetos.

- $\quad$ Con acceso a los datos via Web APIs (REST/SOAP).

- Dispone entre otros más convencionales un sistema de búsqueda RDF -SPARQL (TEN, 2008).

- Una visualización Web del conjunto de objetos.

\section{El proceso de carga de objetos digitales en el repositorio}

El proceso de carga en e-Spacio ha requerido de un cambio de estructura del esquema ims-xml.xsd al FOXML XML schema. Esto se llevó a cabo desarrollando transformaciones xslt cuyo fichero fuente era la descripción según el modelo IEEELOM disponible para cada uno de los objetos y el resultante de la transformación es otro que es directamente cargado en el backend FEDORA.

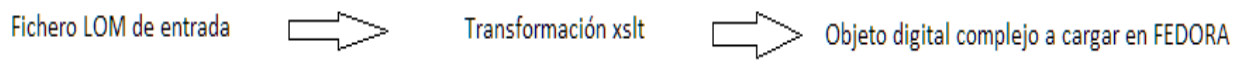

Figura 7. Proceso de tratamiento de metadatos en el repositorio

Los objetos actúan como contenedores de cuatro componentes (datastreams en terminología fedora): uno es el fichero de metadatos LOM, otro con los metadatos dublincore, otro con un fichero $\mathrm{RDF} / \mathrm{xml}$ que da cuenta de las relaciones de ese objeto con otros creados utilizando la ontología indicada anteriormente y un último que da acceso al recurso. 
En la figura 8 se ha representado un aspecto general de la estructura de relaciones basadas en "isMemberOf" mediante la cual se organizan los objetos de aprendizaje y sus componentes. En el bloque de la derecha se observa la disposición de los componentes vinculados a un objeto digital, denominado "Circuito integrador" que a su vez pertenece a una agrupación "Electrónica Analógica” y que a su vez se incorpora en el conjunto de objetos de aprendizaje DIEEC representados en la figura 8.

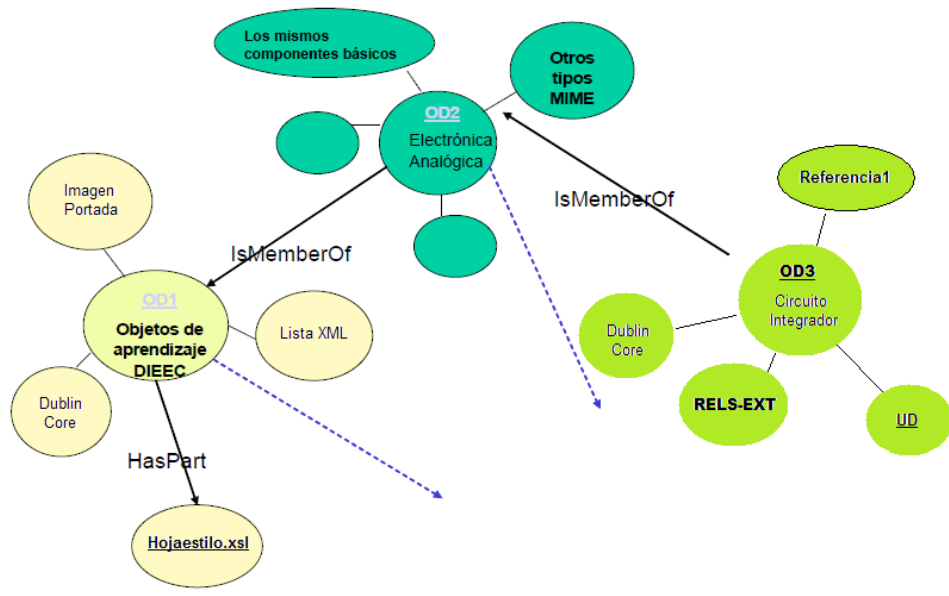

Figura 8. Ejemplo de organización de un objeto digital y sus componentes.

En conclusión, a partir de una descripción LOM se han generado objetos digitales disponibles para ser expuestos en la Web, con identificadores persistentes y todas las funcionalidades añadidas debido a su integración en el repositorio e-Spacio, como búsqueda dentro de ese subconjunto, protocolo OAI_PMH (OAI, 2006], que los incorpora a portales especializados, accesibles mediante Google, exportables en ficheros XML, etc.

\section{Los objetos en la web semántica}

A cada uno de los objetos compuestos se le asignó un identificador único que estaba compuesto de un espacio de nombres oaingeec seguido de la parte específica del objeto que se eligió para que diera algún tipo de información propia del objeto. Por ejemplo: oaingeec:eca-analog-teor-1021 nos indicaba que se trata de un (o)bjeto de (a)prendizaje del departamento de (ing)eniería (e)léctrica y (e)lectrónica de (c)ontrol correspondiente a la teoría analógica. Este aspecto adquiere especial relevancia si pensamos en estos objetos de aprendizaje no como elementos pertenecientes a una 
aplicación sino como elementos de una comunidad accesibles por aplicaciones en la nube.

Pensamos, por tanto, que un aspecto muy relevante de cara a la posibilidad de reutilización y diseminación de estos objetos, sea del total o de alguno de sus componentes, es la posibilidad de su pertenencia a la web semántica. En nuestro caso, cada vez que se crea un objeto se incorpora al almacén RDF una descripción sujeto-predicado-objeto de las diferentes relaciones que se hayan establecido. Es por tanto posible interrogar (sparql) a ese almacén cuyo endpoint está en: e-spacio.uned. es:8080/fedora/risearch.

Al lanzar una pregunta en el endpoint del tipo:

select \$memberCol \$collTitle \$collIdentifier \$member \$memberTitle

from <\#ri>

where

\$memberCol <dc:identifier> 'oaingeec:ing-elec-col-1c4e8f245a1be'

and <info:fedora/oaingeec:ing-elec-col-1c4e8f245a1be> <dc:identifier $>$ \$collidentifier

and \$member <fedora-rels-ext:isMemberOf> <info:fedora/oaingeec:ing-eleccol-1c4e8f245a1be>

and <info:fedora/oaingeec:ing-elec-col-1c4e8f245a1be $><d c$ :title $>$ \$collTitle

and \$member <dc:title> \$memberTitle

da lugar a una respuesta en la web:

$<$ sparql xmlns="http://www.w3.org/2001/sw/DataAccess/rf1/result">

$<$ head $>$

$<$ variable name="memberCol" $/>$

$<$ variable name $=$ "collTitle" $/>$

$<$ variable name $=$ "collidentifier"/>

$<$ variable name $="$ member" $/>$

$<$ variable name $=$ "memberTitle"/>

$</$ head $>$

$<$ results $>$

$<$ result $>$

$<$ memberCol uri="info:fedora/oaingeec:ing-elec-col-1c4e8f245a1be"/>

$<$ collTitle $>$ Par de emisores acoplados $</$ collTitle $>$

$<$ collIdentifier $>$ oaingeec:ing-elec-col-1c4e8f245a1be $</$ collIdentifier $>$

$<$ member uri="info:fedora/oaingeec:eca-analog-teor-10o8"/>

$<$ memberTitle>Amplificador en emisor común con carga pasiva (Requiere Plataforma MicroSim 6.2 ó superior) $</$ memberTitle $>$ 


\section{$</$ result $>$}

$<$ result $>$

$<$ memberCol uri="info:fedora/oaingeec:ing-elec-col-1c4e8f245a1be"/>

$<$ collTitle $>$ Par de emisores acoplados $</$ collTitle $>$

$<$ collIdentifier >oaingeec:ing-elec-col-1c4e8f245a1be</collIdentifier $>$

$<$ member uri="info:fedora/oaingeec:eca-analog-teor-10o9"/>

$<$ memberTitle $>$ Amplificador en emisor común con carga activa. (Requiere

Plataforma MicroSim 6.2 ó superior) $</$ memberTitle $>$

$</$ result $>$

$</$ sparql $>$

que pueda ser entrada para un agente que cree un servicio. Somos conscientes de que para que nuestros objetos sean auténticos linked data y cumplir por tanto uno de los requisitos de la web semántica (incluir enlaces a otras URIs para así profundizar en el descubrimiento de más objetos) que hay que profundizar en la asignación de URIs a elementos tan importantes como autores y materias, pero al menos estamos preparados tecnológicamente para cuando haya algún acuerdo internacional para la asignación de este tipo de identificadores para la Web.

\section{Mostrando los objetos en la Web}

Hemos utilizado la estructura de los objetos de aprendizaje para generar dos vistas en formato HTML. La primera de ellas, representada en la figura 9 ha generado un portal de navegación con arreglo a distintos criterios en http://e-spacio.uned. es:8080/fedora/OAingeec.html. 
L. Zorita; A. López; M. Latorre; M. Blázquez; E. San Cristóbal; S. Martín; G. Díaz; M. Castro CREACIÓN DE OBJETOS DIGITALES DE APRENDIZAJE Y SU INCLUSIÓN EN EL REPOSITORIO INSTITUCIONAL ESPACIO-UNED

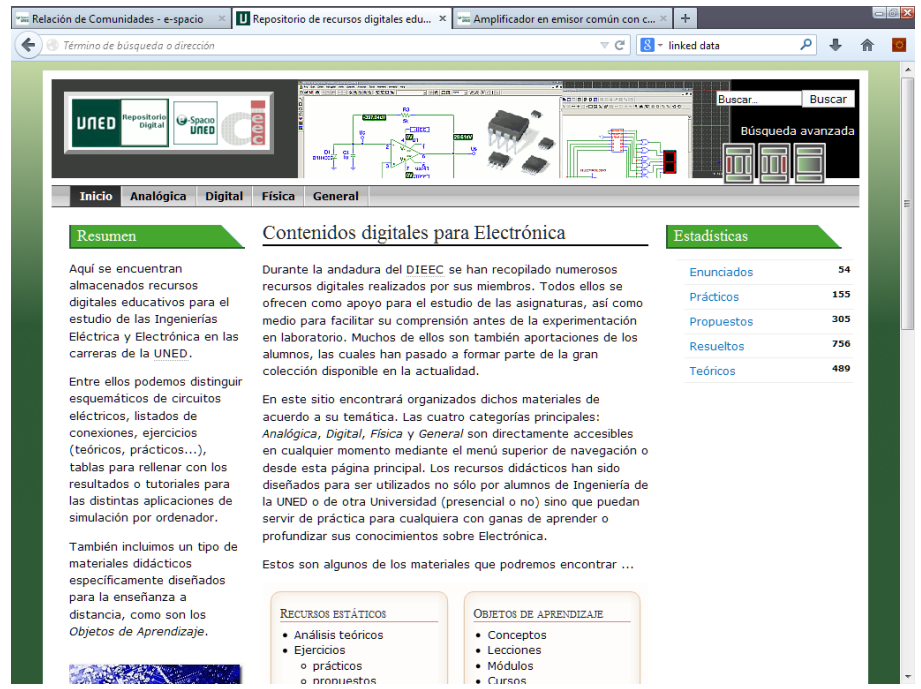

Figura 9. Aspecto del interfaz de localización y acceso a los objetos y contenidos digitales

Por último reseñar que se creó un diseño web para la presentación de estos objetos de aprendizaje basado en transformaciones XSLT sobre la representación XML de los objetos creados, tal y como se puede apreciar en la siguiente figura 10:

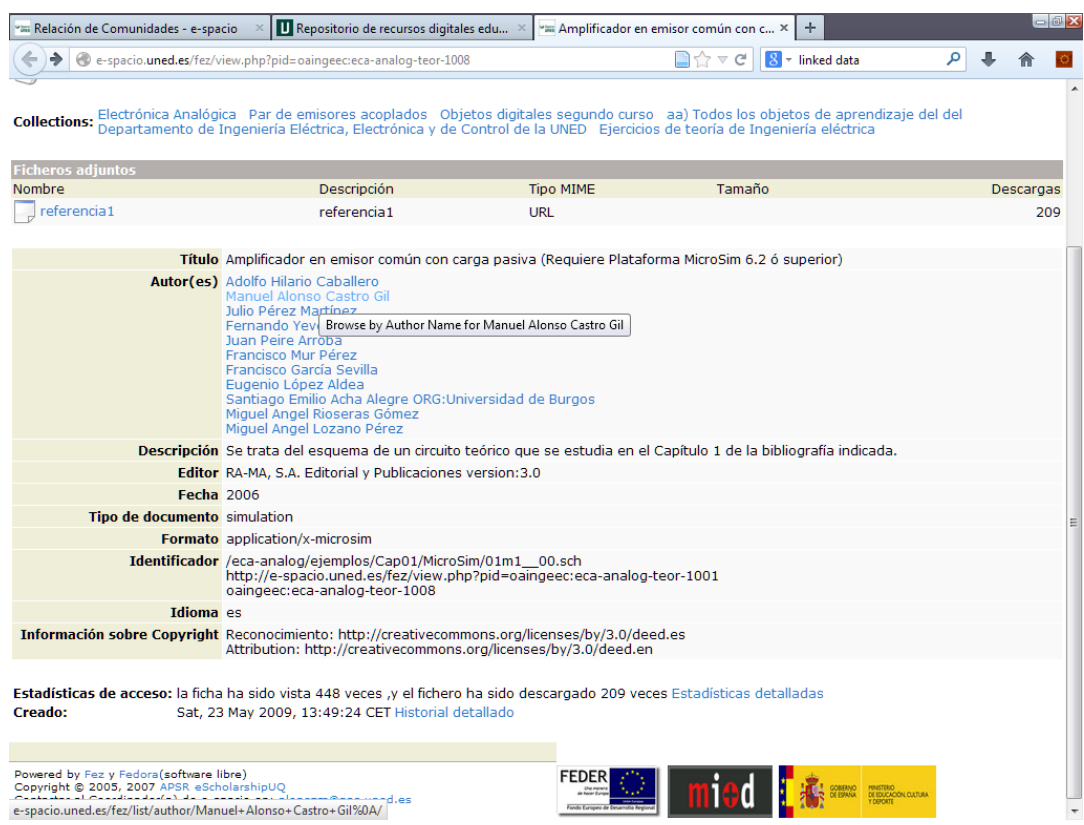

Figura 10. Ficha de objeto digital tras realizar la transformación de metadatos 
y una función asociada que se ajusta al formato de todos los contenidos digitales de e-spacio, que enriquece la navegación y aporta un valor añadido a la información encontrada como la de la figura 11:

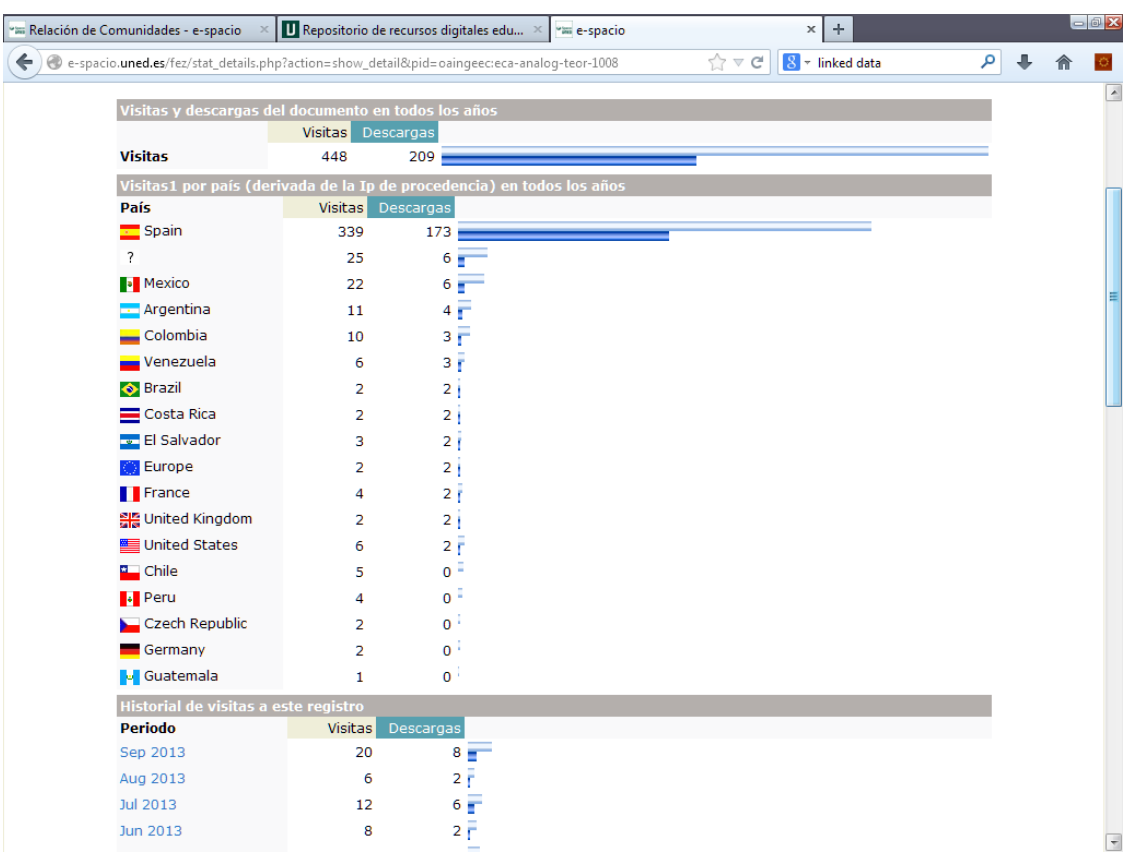

Figura 11. Información de consulta y acceso individualizada para cada objeto digital

\section{CONCLUSIÓN}

A lo largo de este escrito se han descrito los trabajos relacionados con la adquisición y creación de objetos digitales reutilizables obtenidos desde diversas fuentes, los materiales del departamento de Ingeniería Eléctrica, Electrónica y de Control de la UNED y el conocimiento ofrecido por los autores y organizaciones que han tomado parte en los Congresos de Tecnologías Aplicadas para la Enseñanza de la Electrónica.

Se ha descrito profusamente los esfuerzos técnicos por homogeneizar dichos objetos así como para etiquetar y catalogar cada uno de ellos de forma individualizada mediante tecnologías estándares XML de metadatos y tecnologías hechas a medida.

Finalmente, se ha descrito el proceso de carga masiva en el repositorio institucional eSpacio-UNED donde se han alojado miles de objetos digitales con 
sus respectivos documentos de definición de metadatos. Las características del repositorio han hecho posible que todos estos contenidos sirvan para diseminar el conocimiento al mundo a través de la Red.

\section{REFERENCIAS BIBLIOGRÁFICAS}

Ahmed et al. (2001). Professional XML Metadata. Birmingham, Alabama. Wrox Press.

Arriaga, J.; Carpeño, A.; Gordillo, T. (2006). Del objeto de aprendizaje a la implementación de una asignatura. Un caso práctico. Universidad Politécnica de Madrid. Congreso TAEE 2006.

IEEE (2002). Learning Technology Standards Committee of the IEEE. Draft Standard for Learning Object Metadata IEEE 1484.

Marzal García-Quismondo, M. Á.; CalzadaPrado, J.; Cuevas Cerveró, A. (2006). Desarrollo de un esquema de metadatos para la descripción de recursos educativos: El perfil de aplicación MIMETA. Revista española de Documentación Científica, 29, (551-571).

Open Archives Initiative Protocol for Metadata Harvesting (OAI-PMH). (2013). Technologies Report. [en línea] Disponible

en: http://xml.coverpages.org/oams. html [consulta 2013, 26 de octubre].

Proyecto RED OBER (s/f). Objetos Educativos Reutilizables para el EEES en las especialidades de las Tecnologías de la Información y las Comunicaciones. Análisis Estadístico de la Documentación Generada en TAEE (Tecnologías Aplicadas a la Enseñanza de la Electrónica) Proyecto No TSI2007 - 31091 - E. [en línea] Disponible en: http://www. ieec.uned.es/Investigacion/RedOber/ index.htm [consulta 2013, 12 de octubre].

Rouyet, J. I.; Martín, V. (2004). A comparative study of the metadata in SCORM and Dublin Core. Universidad Pontificia de Salamanca.

Ternier, S.; Massart, D.; Campi, A.; Guinea, S.; Ceri, S.; Duval, E. (2008). Interoperability for Searching Learning Object Repositories. The ProLearn Query Language. D-Lib Magazine, 14 (1-2).

\section{PERFIL ACADÉMICO Y PROFESIONAL DE LOS AUTORES}

Luis Zorita trabaja en la Biblioteca de la Universidad Nacional de Educación a Distancia.

E-mail: $\underline{\text { zorita@pas.uned.es }}$

Alicia López Medina trabaja en la Biblioteca de la Universidad Nacional de Educación a Distancia.

E-mail: alopezm@pas.uned.es

Miguel Latorre. Ingeniero Técnico Industrial en la especialidad Electrónica por la Universidad Nacional de Educación a Distancia. Receptor del premio al mejor proyecto fin de carrera 2008-2009 por el Capítulo Español de la Sociedad de Educación del IEEE (CESEI) sobre objetos de aprendizaje. Desarrolla su actividad 
profesional en el Departamento de Ingeniería Eléctrica, Electrónica y de Control de la UNED en el grupo de investigación G-eLios. Student Member del IEEE desde 1999.

\section{E-mail: pelaga@gmail.com}

Manuel Blázquez Merino. Ingeniero Industrial por la Universidad Nacional de Educación a Distancia. Profesor de Enseñanza Secundaria desde 1996 en el área de Tecnología en el Instituto "Ramiro de Maeztu" en Madrid. También es profesor en la Universidad Antonio de Nebrija. Ha sido coeditor de los congresos de educación de Ingeniería EDUCON 2010 y TAEE 2010 y ha escrito cuatro libros de texto para la docencia de Tecnología y diversas guías de estudio.

E-mail: manuel.blazquez.merino@gmail.com

Elio San Cristóbal. Doctor por la UNED e Ingeniero Informático. Actualmente es profesor en la UNED e investiga en el área de tecnologías educativas aplicadas a la docencia de ingeniería, especializado en laboratorios remotos y plataformas educativas.

E-mail: elio@ieec.uned.es

Sergio Martín. Doctor por la UNED e Ingeniero Informático. Actualmente es profesor en la UNED e investiga en el área de tecnologías educativas aplicadas a la docencia de ingeniería, especializado en aplicaciones móviles y laboratorios remotos.

E-mail: smartin@ieec.uned.es

Gabriel Díaz. Doctor en Físicas por la Universidad Autónoma de Madrid. Actualmente es profesor en la UNED e investiga y trabaja en el área de tecnologías educativas aplicadas a la docencia de ingeniería, especializado en seguridad informática y laboratorios remotos.

E-mail: gdiaz@ieec.uned.es

Manuel Castro. Doctor Ingeniero Industrial por la Universidad Politécnica de Madrid. Actualmente es Catedrático en la UNED y Presidente de la Sociedad de la Educación del IEEE. Pertenece al comité organizador delos congresos IEEE EDUCON, IEEE FIE, ISES, TAEE. Es co-editor de IEEE-RITA (Revista Iberoamericana de Tecnologías del Aprendizaje). Es miembro Fellow del IEEE (por sus contribuciones a la enseñanza a distancia en la educación de la ingeniería eléctrica e informática).

E-mail: mcastro@ieec.uned.es 


\section{DIRECCIÓN POSTAL DE LOS AUTORES}

Universidad Nacional de Educación a Distancia

C/ Juan del Rosal 12, 28040, Madrid, España

Fecha de recepción: 07/06/2013

Fecha de aceptación: 15/11/2013

\section{Como citar este artículo:}

Zorita, L.; López Medina, A.; Latorre, M.; Blázquez, M.; San Cristobal, E.; Martín, S.; Díaz, G.; Castro, M. (2014). Creación de objetos digitales de aprendizaje y su inclusión en el repositorio institucional eSpacio-UNED. RIED. Revista Iberoamericana de Educación a Distancia, volumen 17, $\mathrm{n}^{\circ}$ 1, pp. 149-177. 
